\title{
Communication \\ All-Dielectric Metasurface Based on Complementary Split-Ring Resonators for Refractive Index Sensing
}

\author{
Mohsen Samadi ${ }^{1}$ (D), Fatemeh Abshari ${ }^{1}$, José F. Algorri ${ }^{2,3,4}{ }^{(D)}$, Pablo Roldán-Varona ${ }^{2,3,4}{ }^{(D)}$, \\ Luis Rodríguez-Cobo $^{3} \mathbb{D}$, José M. López-Higuera ${ }^{2,3,4}$, José M. Sánchez-Pena ${ }^{5} \mathbb{D}$, Dimitrios C. Zografopoulos ${ }^{6} \mathbb{D}$ \\ and Francesco Dell'Olio ${ }^{7, *(\mathbb{D})}$
}

1 Faculty of Electrical and Computer Engineering, Tarbiat Modares University, Tehran 1411713116, Iran; samadi@physik.uni-kiel.de (M.S.); f.abshari@modares.ac.ir (F.A.)

2 Photonics Engineering Group, University of Cantabria, 39005 Santander, Spain; algorrijf@unican.es (J.F.A.); pablo.roldan@unican.es (P.R.-V.); miguel.lopezhiguera@unican.es (J.M.L.-H.)

3 CIBER-bbn, Instituto de Salud Carlos III, 28029 Madrid, Spain; luis.rodriguez@unican.es

4 Instituto de Investigación Sanitaria Valdecilla (IDIVAL), 39011 Santander, Spain

5 Department of Electronic Technology, Carlos III University, 28911 Madrid, Spain; jmpena@ing.uc3m.es

6 Consiglio Nazionale delle Ricerche, Istituto per la Microelettronica e Microsistemi (CNR-IMM), 00133 Roma, Italy; dimitrios.zografopoulos@artov.imm.cnr.it

7 Department of Electrical and Information Engineering, Polytechnic University of Bari, 70125 Bari, Italy

* Correspondence: francesco.dellolio@poliba.it

check for

updates

Citation: Samadi, M.; Abshari, F.;

Algorri, J.F.; Roldán-Varona, P.;

Rodríguez-Cobo, L.; López-Higuera,

J.M.; Sánchez-Pena, J.M.;

Zografopoulos, D.C.; Dell'Olio, F.

All-Dielectric Metasurface Based on

Complementary Split-Ring

Resonators for Refractive Index

Sensing. Photonics 2022, 9, 130.

https://doi.org/10.3390/

photonics 9030130

Received: 17 January 2022

Accepted: 22 February 2022

Published: 25 February 2022

Publisher's Note: MDPI stays neutral with regard to jurisdictional claims in published maps and institutional affiliations.

Copyright: (C) 2022 by the authors. Licensee MDPI, Basel, Switzerland. This article is an open access article distributed under the terms and conditions of the Creative Commons Attribution (CC BY) license (https:// creativecommons.org/licenses/by/ $4.0 /)$.

\begin{abstract}
Thanks to their lower losses and sharper resonances compared to their metallic counterparts, all-dielectric metasurfaces are attracting a quickly growing research interest. The application of such metasurfaces in the field of refractive index sensing is extremely attractive, especially due to the expected high performance and the simplicity of the sensing element excitation and readout. Herein, we report on an all-dielectric silicon metasurface based on complementary split-ring resonators (CSRRs) optimized for refractive index sensing. A quasi-bound state in the continuum (quasi-BIC) with an ultra-high quality factor can be excited in the near-infrared (NIR) window by violating the structure symmetry. By using the three-dimensional finite element method (3D-FEM), a refractive index sensor for biomedical applications with an ultra-high figure of merit $\left(F o M>100,000 \mathrm{RIU}^{-1}\right)$ has been designed, exploiting the quasi-BIC resonance. The proposed design strategy opens new avenues for developing flat biochemical sensors that are accurate and responsive in real time.
\end{abstract}

Keywords: metasurface; biosensor; bound state in the continuum

\section{Introduction}

Resonant micro- and nano-photonic refractive index sensors have long been utilized for real-time, label-free analysis of chemical and biological samples, such as identifying target biomolecules in a biologic fluid or detecting organic liquid compounds. When target molecules interact with light, the sensor resonance frequency shifts due to light-matter interaction. The frequency shift is subsequently measured and utilized to detect target molecules [1-6]. The sensitivity $\left(S=\Delta \lambda_{\text {res }} / \Delta n\right)$ of refractive index sensors is evaluated as the ratio of the shift in the sensor resonance wavelength to the change in the sample's refractive index. The figure of merit $(F o M=S / F W H M)$ normalizes the refractive index sensitivity to the resonant mode spectral width (full width at the half maximum, FWHM) [5-8].

Surface plasmons [9-12], photonic crystal cavities [3,13-16], and whispering gallery mode resonators $[2,4,17-20]$ have all been used to produce better sensitivities and superior sensing performance. Furthermore, biochemical sensing applications have used plasmonic nanostructures that support Fano resonances [21-24]. However, despite their high sensitivity to the surrounding medium refractive index, they suffer from broad resonances caused by high optical absorption losses in the metal, which severely limit the sensor FoM. 
Metasurfaces (MSs) are planar interfaces made of periodic arrays of sub-wavelength resonant elements used to manipulate the phase, polarization, and amplitude of light [25-27]. They are the 2D equivalents of bulk metamaterials. Due to their lower ohmic losses and thus sharper Fano resonances than their metallic equivalents, all-dielectric MSs have become more popular in recent years for sensing applications [28-34]. In this regard, a new type of MS based on the CSRR [35] was recently reported. In this MS, an ultrathin slot is etched in a silicon layer on a standard glass substrate. In the NIR window, the MS shows two multipolar resonances. Meanwhile, an asymmetry in the structure of the slotted CSRR can be used to trigger a quasi-BIC with an ultra-high Q-factor thanks to the vanishing radiation losses for a small degree of asymmetry. Quasi-BIC modes have already been exploited in many application domains, including nonlinear optics and sensing [36-38].

In our study, by examining the sensitivity of the quasi-BIC mode to the superstrate medium refractive index, we assessed the CSRR-MS sensing capacity. By exploiting the non-radiative nature of the quasi-BIC mode, exceptionally high-quality factors and FoMs can be produced, allowing for the design of highly accurate biological and chemical sensors.

\section{Metasurface}

Figure 1 shows the proposed sensing device. A periodic array of circular slots is fully etched in a silicon layer of thickness $h$, deposited on a glass substrate to form the CSRR-MS. Each unit cell of the periodic structure has one circular slot, whose structural parameters are shown in the inset of Figure 1. The slot width, the inner diameter, the distance between neighboring slots in the $x$-direction, the pitch of the periodic square array, and the size of the silicon gaps are identified as $s, w, g, p$, and $t$, respectively. In addition, an asymmetry parameter $t_{x}$ is introduced in order to break the structure symmetry and excite the quasi-BIC resonance, reducing the arc length in one half of the CSRR structure. The depth of the slots etched in the silicon layer is equal to $h$.

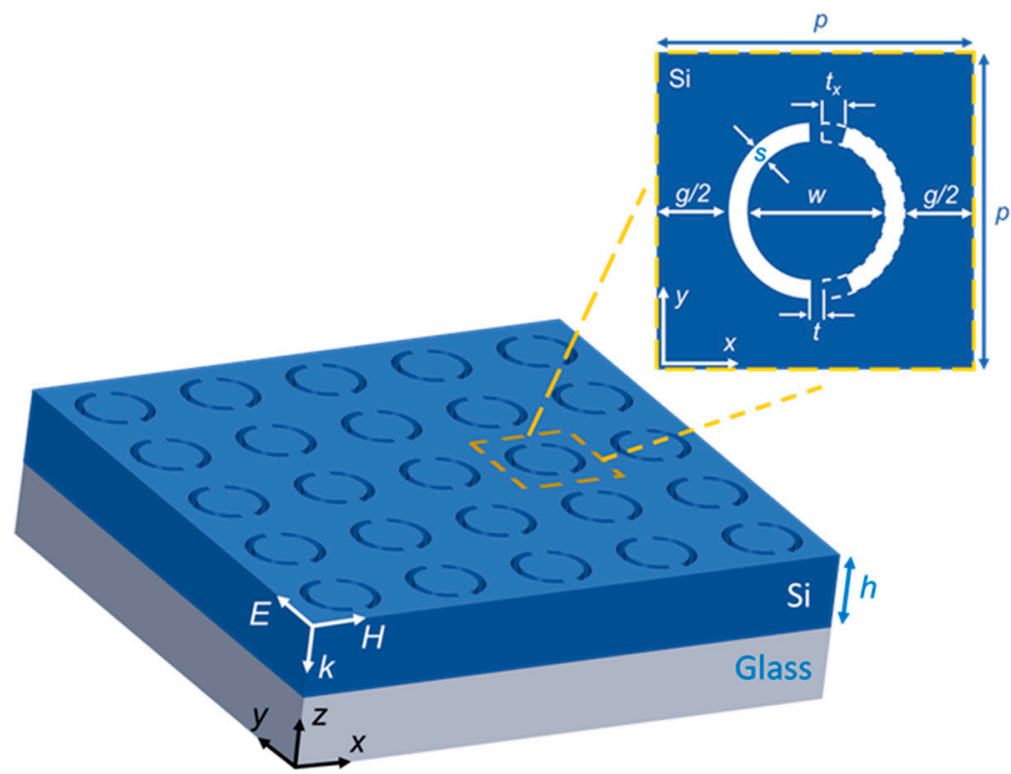

Figure 1. A three-dimensional schematic illustration of the CSRR metasurface. In a silicon layer with a thickness of $h$, the periodic split rings are etched. The unit cell (marked by a yellow dashed square) is shown in the inset.

The transmission spectra of the MS have been computed using the 3D-FEM. The silicon layer is assumed as deposited on a glass substrate with a refractive index of $n_{g}=1.52$, and a $y$-polarized plane wave irradiates the MS in the $z$-direction (see Figure 1). We assume $h=232 \mathrm{~nm}, s=25 \mathrm{~nm}, w=496 \mathrm{~nm}, g=240 \mathrm{~nm}$, and $t=100 \mathrm{~nm}$, which are identical to the values in [35]. The refractive index dispersion of silicon is considered [39]. 
All these geometrical features are compatible with the technological constraints typically imposed by e-beam lithography, having a resolution below $5 \mathrm{~nm}$ [40].

We assume that the metasurface is top illuminated by a sub-pm linewidth laser source operating in the NIR, whose emission frequency can be precisely tuned in a narrow range of a few hundreds of pm by a piezoelectric transducer. A standard detector operating in the NIR can be used for measuring the transmitted power.

\section{Simulation Results}

The MS has been simulated by 3D-FEM. In our simulations, we consider a 1- $\mu$ m-thick glass substrate with the silicon layer on the top. We assume that the Si layer is patterned by a periodic array of 25-nm-wide circular slots. The slots and the $1-\mu \mathrm{m}$-thick volume above the silicon layer are filled with superstrate media of various refractive indices $(n$ in the range 1.31-1.33). The size of each unit cell including one circular slot was $p=786 \mathrm{~nm}$ and periodic boundary conditions were used along $x$ and $y$ directions. Two ports were added on the top and the bottom domains and a $y$-polarized plane wave was incident onto the MS from the top port. The maximum mesh size for the glass substrate, the silicon layer, and the superstrate media was approximately $(\lambda / 7.6),(\lambda / 17.45)$, and $(\lambda / 6.65) \mathrm{nm}$, respectively.

First, we estimated the MS transmission spectra by assuming that the MS was symmetric $\left(t_{x}=0\right)$ and that the etched slots, as well as the volume above the Si layer, were filled with a fluid, as typical for chemosensors and biosensors. In this calculation, we assumed that the fluid had a refractive index $n=1.33$ and neglected the fluid optical absorption. The symmetric MS supports two strong resonances at $\lambda_{1}=1545 \mathrm{~nm}$ and $\lambda_{2}=1610 \mathrm{~nm}$, as shown in Figure 2a. The size of the silicon gap $(t)$ has small effect on these multipolar resonances, as revealed in prior research [35].

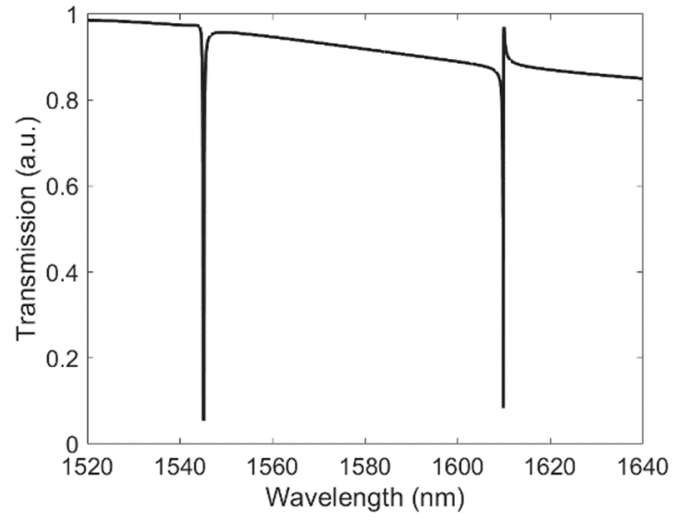

(a)

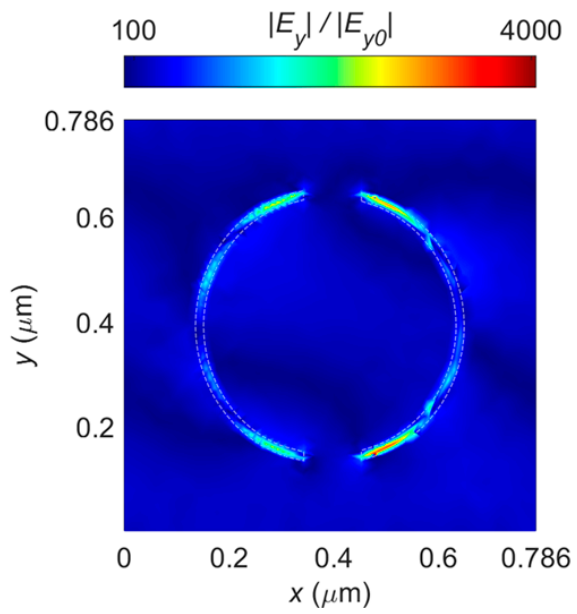

(b)

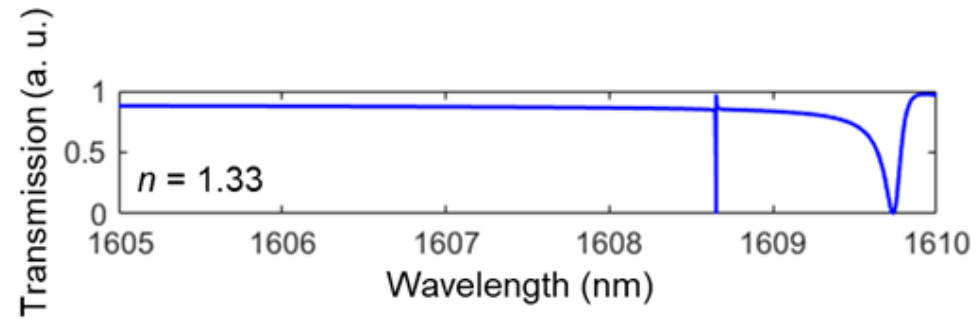

(c)

Figure 2. (a) Transmission spectrum of the symmetric MS $\left(t_{x}=0\right)$. (b) Electric field $\left(\mathrm{E}_{\mathrm{y}}\right)$ distribution relevant to the quasi-BIC resonant mode at the top of the asymmetric MS $\left(t_{x}=10 \mathrm{~nm}\right)$ irradiated in the $z$-direction by a $y$-polarized plane wave. (c) Transmission spectrum of the asymmetric MS $\left(t_{x}=10 \mathrm{~nm}\right.$ ).

In addition to the resonances mentioned above, symmetry breaking allows the described CSRR-MS to support an ultra-high quality factor quasi-BIC mode. We decreased 
the arc length in one half of the CSRR structure by introducing an asymmetry parameter $t_{x}=10 \mathrm{~nm}$ to violate the structure in-plane inversion symmetry and trigger the quasi-BIC mode, as shown in [35]. The in-plane electric field $\left(E_{y}\right)$ of the quasi-BIC resonant mode was calculated at the top surface of the asymmetric CSRR MS $\left(t_{x}=10 \mathrm{~nm}\right)$ and plotted in Figure $2 \mathrm{~b}$. The transmission spectrum of the asymmetric MS in the narrow wavelength ranging from $1605 \mathrm{~nm}$ to $1610 \mathrm{~nm}$ is shown in Figure 2c, considering again $n=1.33$ and $k=0$ ( $k$ is the extinction coefficient of the superstrate medium). The quasi-BIC resonance occurs at $\lambda_{\text {res }}=1608.7 \mathrm{~nm}$.

To study the asymmetric MS performance as a refractive index sensor, we varied the refractive index $n$ of the fluid inside the etched slots and above the Si layer in the range from 1.31 to 1.33 . Then, the transmission spectrum in each case was calculated to investigate the sensitivity of the quasi-BIC mode to $n$. Figure 3 shows the outcome of our numerical calculations. The dependence of the resonance wavelength on $n$ is almost linear, as demonstrated in the linear fit of the dashed line in Figure 3.

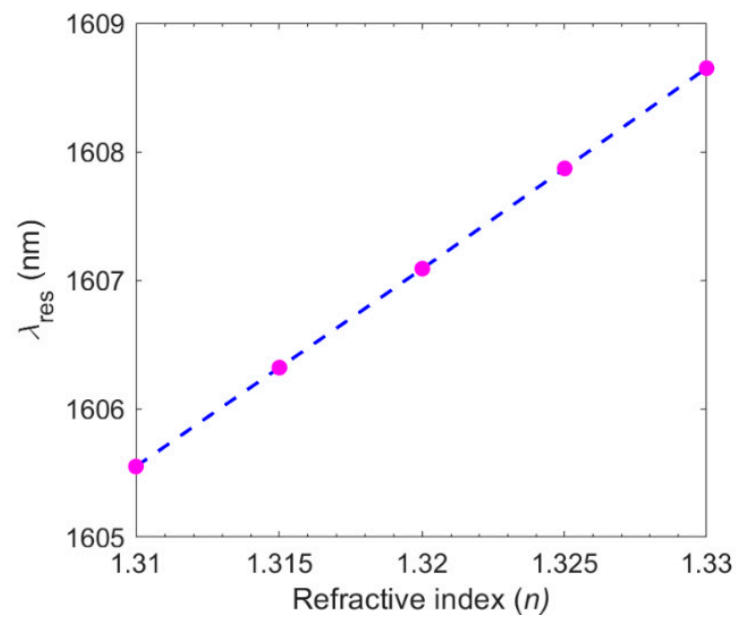

Figure 3. Dependence of the resonance wavelength on $n$, i.e., the refractive index of the aqueous medium inside the etched slots and above the Si layer. The CSRR-MS is asymmetric, with $t_{x}=10 \mathrm{~nm}$.

The sensitivity of the quasi-BIC mode to changes in the superstrate medium refractive index $\left(S=\lambda_{\text {res }} / n\right)$ was computed and found to be $S=155 \mathrm{~nm} \mathrm{RIU}^{-1}$. In addition to $S$, we considered the figure of merit $(F o M)$, defined as $F o M=S / F W H M$, where FWHM is the full width at half maximum of the transmission drop at resonance wavelength. For example, for the quasi-BIC mode at $=1608.7 \mathrm{~nm}$, we estimated a FWHM of $0.4 \mathrm{pm}$, resulting in an ultra-high FoM of $387.500 \mathrm{RIU}^{-1}$. Although the archived value of $S$ is comparable to that reported for other all-dielectric metasurfaces [34] and worse than that obtainable with some plasmonic sensors [41], we stress that the main performance parameter is the FoM, whose value exceeds $10^{5}$.

We evaluated the effect of a nonzero value of $k$ on the quasi-BIC resonance and the FWHM. We calculated the transmission spectra of the asymmetric CSRR MS $\left(t_{x}=10 \mathrm{~nm}\right)$ for a constant superstrate refractive index of $n=1.33$ and different values of the superstrate extinction coefficient ( $k$ ranging from 0 to $5 \times 10^{-6}$ ). The FWHM of the resonance was increased for higher $k$ values due to larger optical losses inside the superstrate medium, leading to a low-FoM sensing device, as shown by Figure $4 \mathrm{a}$. Figure $4 \mathrm{~b}$ shows that the FWHM of the quasi-BIC mode changes non-linearly with variations of the superstrate extinction coefficient $(k)$. While the value of FWHM is nearly constant for $k<10^{-6}$, a sharp increase was observed for larger $k$ values. 


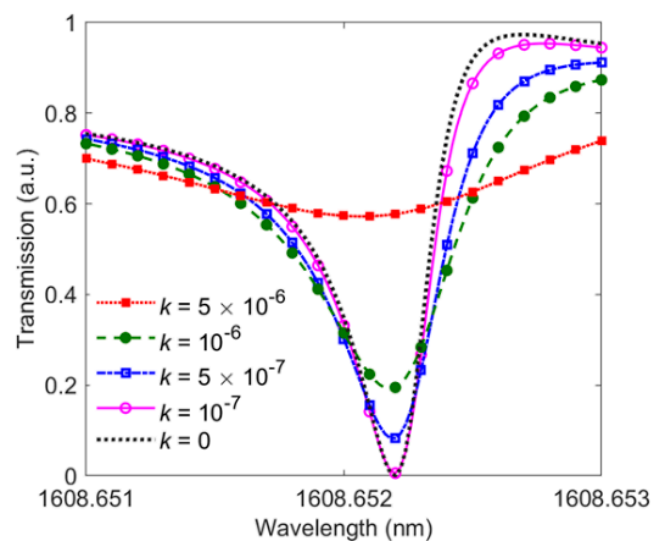

(a)

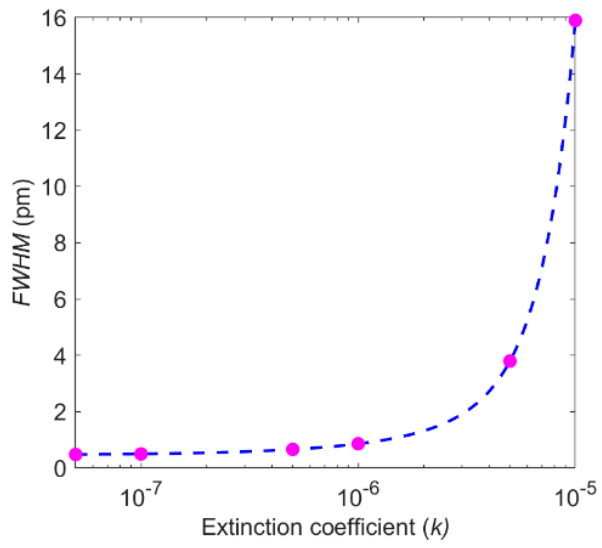

(b)

Figure 4. (a) Resonance relevant to the quasi-BIC mode for several values of $k$. (b) FWHW of the quasi-BIC mode vs. $k$.

Since the values of $k$ are below $10^{-6}$ for several applications involving, for example, organic liquid compound detection [42], we expect a modest degradation of the FoM (reduction factor $<2$ ) due to the nonzero values of $k$ when applications involving fluids having $k<10^{-6} @ 1.6 \mu \mathrm{m}$ are considered.

\section{Conclusions}

We report on the design of a refractive index sensor with an ultra-high figure of merit $\left(>10^{5} \mathrm{RIU}^{-1}\right)$ based on all-dielectric metasurfaces that support a quasi-BIC mode. Our 3D numerical simulations showed that the ultra-narrow quasi-BIC resonance in the complementary split-ring resonator metasurface structure can attain a sensitivity value of $155 \mathrm{~nm} \mathrm{RIU}^{-1}$ and an outstanding FoM of $387,500 \mathrm{RIU}^{-1}$, when $k=0$. This high value of $F o M$ is obtained with a value of $t_{x}$ (the geometrical asymmetry parameter) equal to $10 \mathrm{~nm}$, which is feasible from a technological point of view. Considering new biomedical applications, the proposed technique offers new paths of research on light-matter interactions. In addition, cluster analysis could be utilized to enhance the resolution of chemosensors and biosensors developed according to the approach discussed here [43,44].

Author Contributions: Conceptualization, M.S. and F.D.; methodology, M.S., F.A. and F.D.; software, M.S. and F.A.; validation, J.F.A., P.R.-V., L.R.-C. and D.C.Z.; investigation, M.S., F.A. and J.F.A.; writing — original draft preparation, M.S. and F.D.; writing—-review and editing, all authors; supervision, D.C.Z. and F.D.; project administration, J.M.L.-H. and J.M.S.-P.; funding acquisition, J.M.S.-P. and F.D. All authors have read and agreed to the published version of the manuscript.

Funding: This work is part of the projects PID2019-107270RB-C21 and PID2019-109072RB-C31, funded by MCIN/AEI/10.13039/501100011033 and FEDER "A way to make Europe", PDC2021121172-C21, funded by MCIN/AEI/10.13039/501100011033 and European Union "Next generation EU"/PTR, TeDFeS Project (RTC-2017-6321-1 funded by MCIN/AEI/10.13039/501100011033 and FEDER "A way to make Europe"), and project S2018/NMT-4326, funded by the Comunidad de Madrid and FEDER Program. J.F.A. received funding from Ministerio de Ciencia, Innovación y Universidades of Spain under the Juan de la Cierva-Incorporación grant.

Institutional Review Board Statement: Not applicable.

Informed Consent Statement: Not applicable.

Data Availability Statement: Not applicable.

Conflicts of Interest: The authors declare no conflict of interest. 


\section{References}

1. Arnold, S.; Khoshsima, M.; Teraoka, I.; Holler, S.; Vollmer, F. Shift of Whispering-Gallery Modes in Microspheres by Protein Adsorption. Opt. Lett. 2003, 28, 272-274. [CrossRef] [PubMed]

2. Yalcin, A.; Popat, K.C.; Aldridge, J.C.; Desai, T.A.; Hryniewicz, J.; Chbouki, N.; Little, B.E.; Oliver, K.; Van, V.; Chu, S.; et al. Optical Sensing of Biomolecules Using Microring Resonators. IEEE J. Sel. Top. Quantum Electron. 2006, 12, 148-155. [CrossRef]

3. Lee, M.R.; Fauchet, P.M. Two-Dimensional Silicon Photonic Crystal Based Biosensing Platform for Protein Detection. Opt. Express 2007, 15, 4530. [CrossRef] [PubMed]

4. Zhu, H.; White, I.M.; Suter, J.D.; Dale, P.S.; Fan, X. Analysis of Biomolecule Detection with Optofluidic Ring Resonator Sensors. Opt. Express 2007, 15, 9139. [CrossRef]

5. Homola, J. Surface Plasmon Resonance Sensors for Detection of Chemical and Biological Species. Chem. Rev. 2008, 108, 462-493. [CrossRef]

6. Spackova, B.; Wrobel, P.; Bockova, M.; Homola, J. Optical Biosensors Based on Plasmonic Nanostructures: A Review. Proc. IEEE 2016, 104, 2380-2408. [CrossRef]

7. Mayer, K.M.; Hafner, J.H. Localized Surface Plasmon Resonance Sensors. Chem. Rev. 2011, 111, 3828-3857. [CrossRef]

8. Xu, Y.; Bai, P.; Zhou, X.; Akimov, Y.; Png, C.E.; Ang, L.; Knoll, W.; Wu, L. Optical Refractive Index Sensors with Plasmonic and Photonic Structures: Promising and Inconvenient Truth. Adv. Opt. Mater. 2019, 7, 1801433. [CrossRef]

9. Chen, H.; Kou, X.; Yang, Z.; Ni, W.; Wang, J. Shape- and Size-Dependent Refractive Index Sensitivity of Gold Nanoparticles. Langmuir 2008, 24, 5233-5237. [CrossRef]

10. Svedendahl, M.; Chen, S.; Dmitriev, A.; Käll, M. Refractometric Sensing Using Propagating versus Localized Surface Plasmons: A Direct Comparison. Nano Lett. 2009, 9, 4428-4433. [CrossRef]

11. Shalabney, A.; Abdulhalim, I. Figure-of-Merit Enhancement of Surface Plasmon Resonance Sensors in the Spectral Interrogation. Opt. Lett. 2012, 37, 1175. [CrossRef] [PubMed]

12. Lodewijks, K.; Van Roy, W.; Borghs, G.; Lagae, L.; Van Dorpe, P. Boosting the Figure-Of-Merit of LSPR-Based Refractive Index Sensing by Phase-Sensitive Measurements. Nano Lett. 2012, 12, 1655-1659. [CrossRef] [PubMed]

13. Nair, R.V.; Vijaya, R. Photonic Crystal Sensors: An Overview. Prog. Quantum Electron. 2010, 34, 89-134. [CrossRef]

14. Guo, Y.; Ye, J.Y.; Divin, C.; Huang, B.; Thomas, T.P.; Baker, J.R., Jr.; Norris, T.B. Real-Time Biomolecular Binding Detection Using a Sensitive Photonic Crystal Biosensor. Anal. Chem. 2010, 82, 5211-5218. [CrossRef]

15. Baker, J.E.; Sriram, R.; Miller, B.L. Two-Dimensional Photonic Crystals for Sensitive Microscale Chemical and Biochemical Sensing. Lab Chip 2015, 15, 971-990. [CrossRef] [PubMed]

16. Zhang, Y.; Zhao, Y.; Lv, R. A Review for Optical Sensors Based on Photonic Crystal Cavities. Sens. Actuators Phys. 2015, 233, 374-389. [CrossRef]

17. Baehr-Jones, T.; Hochberg, M.; Walker, C.; Scherer, A. High-Q Ring Resonators in Thin Silicon-on-Insulator. Appl. Phys. Lett. 2004, 85, 3346-3347. [CrossRef]

18. Ksendzov, A.; Lin, Y. Integrated Optics Ring-Resonator Sensors for Protein Detection. Opt. Lett. 2005, 30, 3344. [CrossRef]

19. Armani, A.M.; Kulkarni, R.P.; Fraser, S.E.; Flagan, R.C.; Vahala, K.J. Label-Free, Single-Molecule Detection with Optical Microcavities. Science 2007, 317, 783-787. [CrossRef]

20. Zhu, J.; Ozdemir, S.K.; Xiao, Y.-F.; Li, L.; He, L.; Chen, D.-R.; Yang, L. On-Chip Single Nanoparticle Detection and Sizing by Mode Splitting in an Ultrahigh-Q Microresonator. Nat. Photonics 2010, 4, 46-49. [CrossRef]

21. Liu, S.-D.; Yang, Z.; Liu, R.-P.; Li, X.-Y. High Sensitivity Localized Surface Plasmon Resonance Sensing Using a Double Split NanoRing Cavity. J. Phys. Chem. C 2011, 115, 24469-24477. [CrossRef]

22. Fu, Y.H.; Zhang, J.B.; Yu, Y.F.; Luk'yanchuk, B. Generating and Manipulating Higher Order Fano Resonances in Dual-Disk Ring Plasmonic Nanostructures. ACS Nano 2012, 6, 5130-5137. [CrossRef] [PubMed]

23. Zhang, Q.; Wen, X.; Li, G.; Ruan, Q.; Wang, J.; Xiong, Q. Multiple Magnetic Mode-Based Fano Resonance in Split-Ring Resonator/Disk Nanocavities. ACS Nano 2013, 7, 11071-11078. [CrossRef] [PubMed]

24. Zhan, Y.; Lei, D.Y.; Li, X.; Maier, S.A. Plasmonic Fano Resonances in Nanohole Quadrumers for Ultra-Sensitive Refractive Index Sensing. Nanoscale 2014, 6, 4705-4715. [CrossRef] [PubMed]

25. Kildishev, A.V.; Boltasseva, A.; Shalaev, V.M. Planar Photonics with Metasurfaces. Science 2013, 339, 1232009. [CrossRef]

26. Yu, N.; Capasso, F. Flat Optics with Designer Metasurfaces. Nat. Mater. 2014, 13, 139-150. [CrossRef]

27. Genevet, P.; Capasso, F.; Aieta, F.; Khorasaninejad, M.; Devlin, R. Recent Advances in Planar Optics: From Plasmonic to Dielectric Metasurfaces. Optica 2017, 4, 139. [CrossRef]

28. Shen, Z.; Du, M. High-Performance Refractive Index Sensing System Based on Multiple Fano Resonances in PolarizationInsensitive Metasurface with Nanorings. Opt. Express 2021, 29, 28287. [CrossRef]

29. Romano, S.; Zito, G.; Torino, S.; Calafiore, G.; Penzo, E.; Coppola, G.; Cabrini, S.; Rendina, I.; Mocella, V. Label-Free Sensing of Ultralow-Weight Molecules with All-Dielectric Metasurfaces Supporting Bound States in the Continuum. Photonics Res. 2018, 6, 726. [CrossRef]

30. Zhang, Y.; Liu, W.; Li, Z.; Li, Z.; Cheng, H.; Chen, S.; Tian, J. High-Quality-Factor Multiple Fano Resonances for Refractive Index Sensing. Opt. Lett. 2018, 43, 1842. [CrossRef]

31. Algorri, J.; Zografopoulos, D.; Ferraro, A.; García-Cámara, B.; Vergaz, R.; Beccherelli, R.; Sánchez-Pena, J. Anapole Modes in Hollow Nanocuboid Dielectric Metasurfaces for Refractometric Sensing. Nanomaterials 2018, 9, 30. [CrossRef] [PubMed] 
32. Leitis, A.; Tittl, A.; Liu, M.; Lee, B.H.; Gu, M.B.; Kivshar, Y.S.; Altug, H. Angle-Multiplexed All-Dielectric Metasurfaces for Broadband Molecular Fingerprint Retrieval. Sci. Adv. 2019, 5, eaaw2871. [CrossRef] [PubMed]

33. Su, W.; Ding, Y.; Luo, Y.; Liu, Y. A High Figure of Merit Refractive Index Sensor Based on Fano Resonance in All-Dielectric Metasurface. Results Phys. 2020, 16, 102833. [CrossRef]

34. Tognazzi, A.; Rocco, D.; Gandolfi, M.; Locatelli, A.; Carletti, L.; De Angelis, C. High Quality Factor Silicon Membrane Metasurface for Intensity-Based Refractive Index Sensing. Optics 2021, 2, 193-199. [CrossRef]

35. Algorri, J.F.; Dell'Olio, F.; Roldán-Varona, P.; Rodríguez-Cobo, L.; López-Higuera, J.M.; Sánchez-Pena, J.M.; Zografopoulos, D.C. Strongly Resonant Silicon Slot Metasurfaces with Symmetry-Protected Bound States in the Continuum. Opt. Express 2021, 29, 10374. [CrossRef]

36. Gandolfi, M.; Tognazzi, A.; Rocco, D.; De Angelis, C.; Carletti, L. Near-Unity Third-Harmonic Circular Dichroism Driven by a Quasibound State in the Continuum in Asymmetric Silicon Metasurfaces. Phys. Rev. A 2021, 104, 023524. [CrossRef]

37. Romano, S.; Zito, G.; Lara Yépez, S.N.; Cabrini, S.; Penzo, E.; Coppola, G.; Rendina, I.; Mocellaark, V. Tuning the Exponential Sensitivity of a Bound-State-in-Continuum Optical Sensor. Opt. Express 2019, 27, 18776. [CrossRef]

38. Algorri, J.F.; Dell'Olio, F.; Roldán-Varona, P.; Rodríguez-Cobo, L.; López-Higuera, J.M.; Sánchez-Pena, J.M.; Dmitriev, V.; Zografopoulos, D.C. Analogue of Electromagnetically Induced Transparency in Square Slotted Silicon Metasurfaces Supporting Bound States in the Continuum. Opt. Express 2022, 30, 4615. [CrossRef]

39. Green, M.A. Self-Consistent Optical Parameters of Intrinsic Silicon at 300K Including Temperature Coefficients. Sol. Energy Mater. Sol. Cells 2008, 92, 1305-1310. [CrossRef]

40. Altissimo, M. E-Beam Lithography for Micro-/Nanofabrication. Biomicrofluidics 2010, 4, 026503. [CrossRef]

41. Pitruzzello, G.; Krauss, T.F. Photonic Crystal Resonances for Sensing and Imaging. J. Opt. 2018, 20, 073004. [CrossRef]

42. Scheurich, S.; Belle, S.; Hellmann, R.; So, S.; Sparrow, I.J.G.; Emmerson, G. Application of a Silica-on-Silicon Planar Optical Waveguide Bragg Grating Sensor for Organic Liquid Compound Detection; Baldini, F., Homola, J., Lieberman, R.A., Eds.; International Society for Optics and Photonics: Prague, Czech Republic, 2009; p. 73561B.

43. Peli, S.; Ronchi, A.; Bianchetti, G.; Rossella, F.; Giannetti, C.; Chiari, M.; Pingue, P.; Banfi, F.; Ferrini, G. Optical and Mechanical Properties of Streptavidin-Conjugated Gold Nanospheres through Data Mining Techniques. Sci. Rep. 2020, 10, 16230. [CrossRef] [PubMed]

44. Ronchi, A.; Sterzi, A.; Gandolfi, M.; Belarouci, A.; Giannetti, C.; Fatti, N.D.; Banfi, F.; Ferrini, G. Discrimination of Nano-Objects via Cluster Analysis Techniques Applied to Time-Resolved Thermo-Acoustic Microscopy. Ultrasonics 2021, 114, 106403. [CrossRef] [PubMed] 